\title{
CLINICAL MANIFESTATIONS OF SECONDARY APLASIA AFTER CHEMOTHERAPY
}

\author{
Erzsebet Papp Zsuzsanna, Maria-Adriene Horvath \\ Pediatric Department, University of Medicine and Pharmacy, Targu-Mures \\ Mures County Hospital, Targu-Mures
}

\begin{abstract}
Objectives. Our aims were to highlight the clinical signs and symptoms of secondary bone marrow aplasia after intensive chemotherapy.

Material and methods. We included in the study group 20 children with intensive cytostatic treatment aged between 9 months and 18 years, all the patients were hospitalized in the hemato-oncology department of the Pediatric Clinic Targu-Mures with the following diagnoses: acute lymphoblastic leukemia, Wilms tumor, acute myelogenous leukemia, Hodgkin lymphoma, non-Hodgkin lymphoma, neuroblastoma, and round small cell desmoplastic tumor. The inclusion criteria was the intensive chemotherapy, exclusion criteria was maintenance cytostatic treatment. It was made a dynamic clinical-biological observation protocol to note the clinical considerations of the aplastic periods after each cytostatic treatment. Bone marrow aplasia was certified by the neutropenia, anemia and thrombocytopenia.

Results and discussions. The majority pathology in our study group was lymphoblastic acute leukemia (60\%), followed by Wilms tumor, and one case of acute myelogenous leukemia, Hodgkin lymphoma, non-Hodgkin lymphoma, neuroblastoma, and round small cell desmoplastic tumor the distribution of age groups respecting literature data. All the patients were treated with international protocols, and after each cure followed the secondary bone marrow aplasia with the afferent pathologies: anemia, thrombocytopenia, mucositis, infections with different localizations, zona zoster reactivation.

Conclusions. Secondary bone marrow aplasia duration and severity differ by cancer type. We observed the most severe clinical complications in the ALL and AML patients. Infectious complications have evolved with the individualized shades probably generated by immunological peculiarities.
\end{abstract}

Keywords: aplasia, child, chemotherapy, cancer

\section{INTRODUCTION}

Childhood cancer is an extremely rare pathology, representing only $2 \%$ of pediatric pathology and about $2 \%$ of all cancer diseases. Annually 200,000 pediatric cancers occur and in Romania there are detected around 500 cases (1).

Currently 5,000 children are registered with a diagnosis of malignancy undergoing intense chemotherapy treatment or are in remission (2). Pediatric cancers present a specific incidence profile: leukemias and lymphomas in first place, followed by cerebral tumors - second place, after them neuroblastoma, nephroblastoma (Wilms tumor), retinoblastoma, hepatoblastoma, rhabdomyosarcoma. (3)
Therapeutic arsenal varies depending on the diagnosis and consists of chemotherapy, surgical solving, radiotherapy and bone marrow transplantation- treatment should always be chosen based on well-established international protocols. Overall survival in pediatric oncology is described in the literature as about $80 \%$ in certain pathologies by referring even more positive (ALL, WT) (4) but still are some diseases where the prognosis remains around 50\% (LAM, NB). (1)

Chemotherapy aims to destroy cancer cells and one of the most important side effects is aplasia, when the bone marrow does not produce precursors of the three major cell lines leukocytes, erythrocytes and platelets. From these lacks will follow the

Corresponding author:

Erzsebet Papp Zsuzsanna, Mures County Hospital, 38 Gheorghe Marinescu Street, Targu-Mures

E-mail: pappzsuzsi@freemail.hu 
many complications associated with signs and symptoms that may endanger patients' lives.

\section{MATERIAL AND METHODS}

We included in the study group 20 children with intensive cytostatic treatment, all the patients were hospitalized in the hemato-oncology department of the Pediatric Clinic Targu-Mures with the following diagnoses: acute lymphoblastic leukemia, Wilms tumor, acute myelogenous leukemia, Hodgkin lymphoma, non-Hodgkin lymphoma, neuroblastoma, and round small cell desmoplastic tumor. The inclusion criteria was the intensive chemotherapy, exclusion criteria was maintenance cytostatic treatment. It was made a dynamic clinical-biological observation protocol to note the clinical considerations of the aplastic periods after each cytostatic treatment. Bone marrow aplasia was certified by the neutropenia, anemia and thrombocytopenia.

\section{RESULTS}

In the study were enrolled 20 children (9 girls, 11 boys ) with intense chemotherapy treatment.

Majority pathology was preB cell LAL (60\%), then followed 3 cases of WT ( 1 case of pulmonary metastasis), and one case of M2 AML, LH, Non Hodgkin lymphoma, stage 4 small round cell desmoplastic tumor with liver metastases, treated and relapsed presacral neuroblastoma.

(Fig. 1) The age of children ranged from 9 months to 18 years (mean -7.18 ). (Fig. 2)

All of our patients were treated according international Protocolls, so those with LAL - ALL BFM 2002 ( 1 case HR Cr. Ph + associated with Imatinib) and the 9 month old infant with Interfant 99, AMLAML BFM 2002, LH-HD DAL 90, LNH-ALCL international protocol 2000, WT-SIOP Nefroblastoma 2000, NB-SIOPEN VP/CARBO+CADO, Desmoplastic round small cell tumor - CWS 2009.

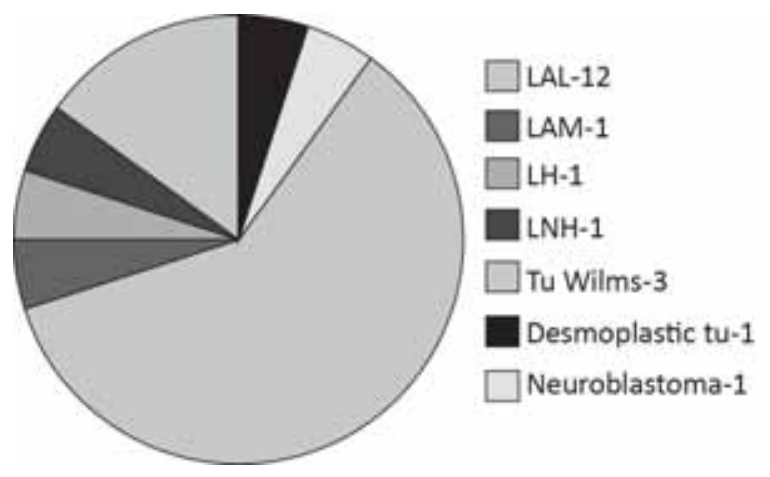

FIGURE 1. Phatology distribution

Of the ALL patients 4 were enrolled in high risk based on immunofenotyping, CNS damage, the presence of Philadelphia chromosome or the response to prednisone on day 8 of treatment. Solid tumors were diagnosed in advanced stages 3-4, which made it difficult therapeutic approach.

After each chemotherapy block followed a period of aplasia. The average duration of periods of aplasia in children with leukemia was significantly longer -15.23 days than in those with solide tumors -4.71 days $(\mathrm{p}<0.0001)-$ Fig. 3 .

The extreme blood values varied between $\mathrm{Hgb}$ 5-7 (LAL/LNH) Valorile hematologice extreme găsite au variat între $\mathrm{Hgb} 5 \mathrm{~g} / \mathrm{dl}$ (LAL) and $7 \mathrm{~g} / \mathrm{dl}$ (LNH), Leucocytea 20/mmc (LAL) and 450/mmc (NB), Trombocytes $2,500 / \mathrm{mmc}$ (LAL) and 20,000/ mmc (NB) - Fig. 4.

At low levels of Hgb no child reported subjective complaints, only clinical skin pallor was observed.

Thrombocytopenia in 3 cases with ALL caused major bleeding incidents (menometrorrhage, HDI, repeated epistaxis) and in 1 child with Wilms Tumor inferior digestive hemorrhage.

Purpura and ecchymosis were observed in 3 children with ALL.

Leukopenia was complicated by infection in all children with acute leukemia and solid tumors except for the 18 years old patient with round -small

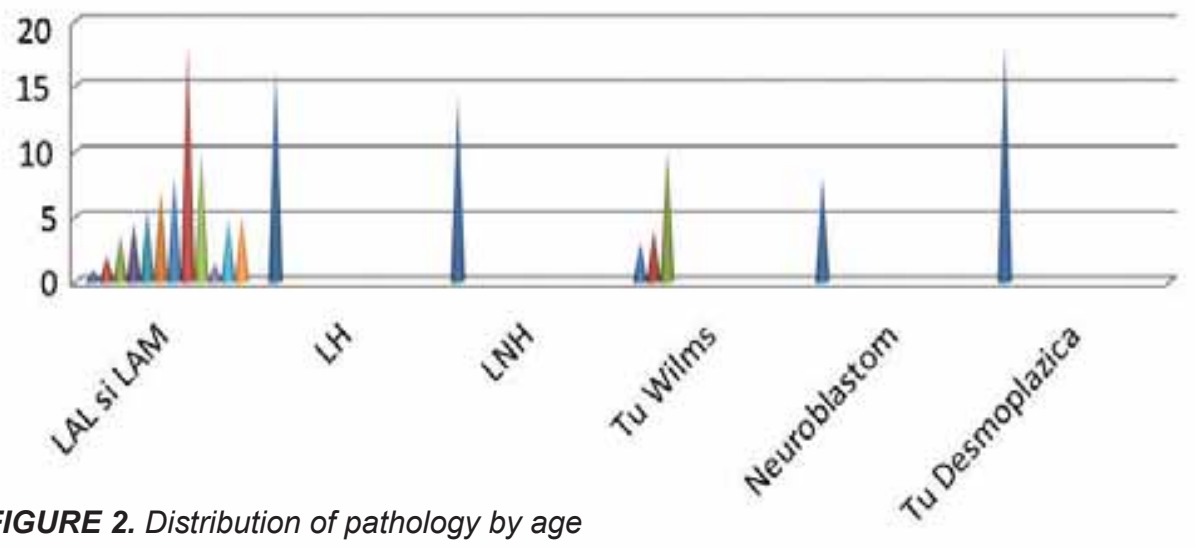




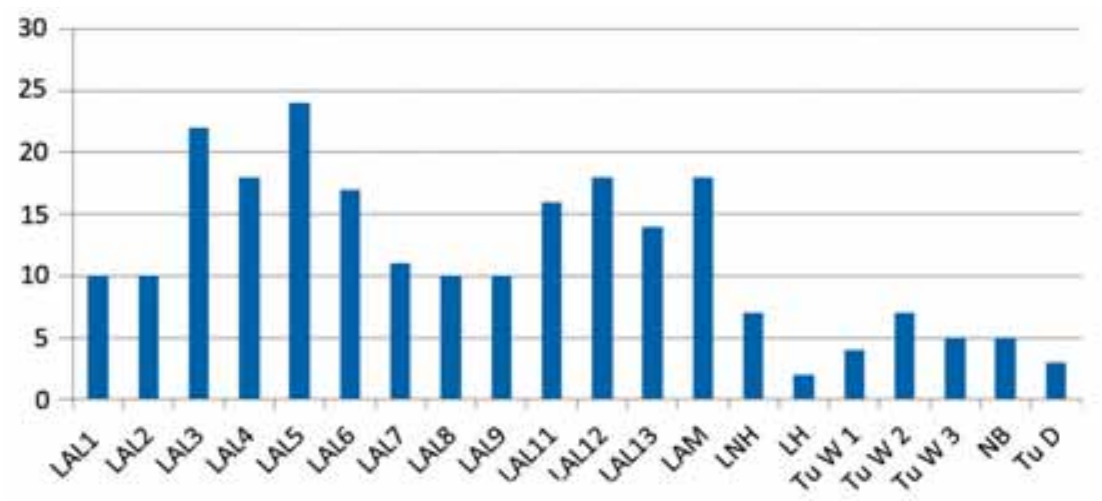

FIGURE 3. Mean duration of aplastic periods

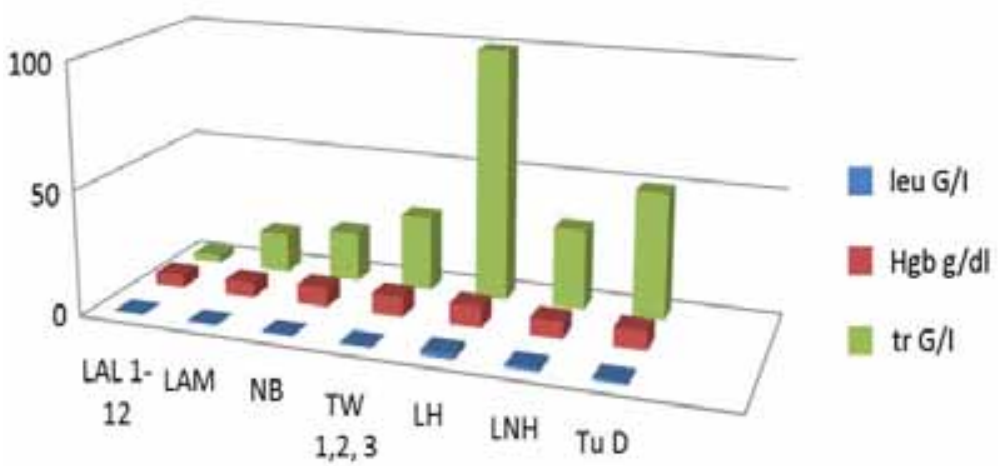

FIGURE 4. Hematologic parameters - extreme values

cell desmoplastic tumor (Fig. 5). All children with leukemia (13) and the non Hodgkin lymphoma patient while leukocytes were decreasing presented oral lesions (mucositis sores) which have become infected causing ulcers. (Fig. 6, 7) Cultures taken from the injuries did not come out positive. All subjective complaints were severe pain when chewing, food refusal.

Next frequent pathology, the bacterial pneumonia was manifested in 8 children with ALL, AML patient, 2 of children with WT, in patients with NHL and NB.

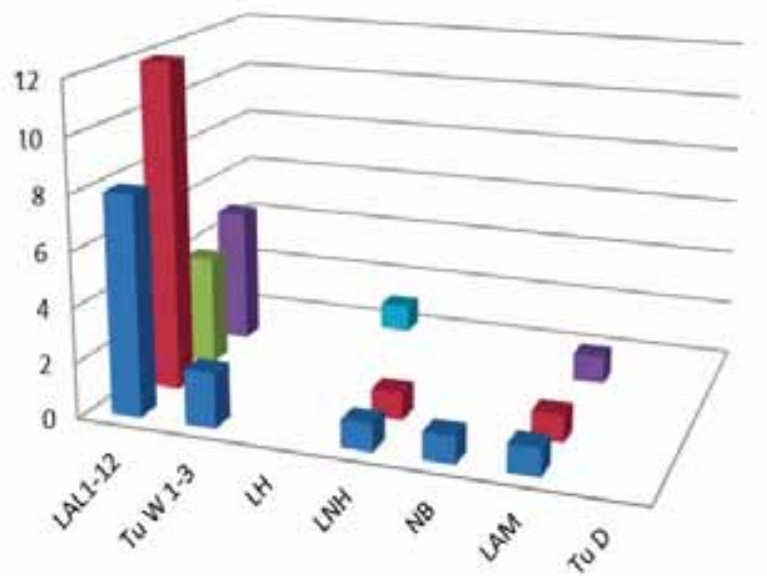

- bacterial pneumonia

infected mucositis

II panaritium

enteral infections

zona zoster
Clinical signs were cough, fever, dyspnoea Treatments consisted of associated antibiotics (CG2-3, Meropenem, antistaphylococcal treatment) and immune support by intravenous immunoglobulin.

In a patient aged 12 years with $\mathrm{Ph}$ cr. positive ALL, after a course of aggressive chemotherapy (8th month of treatment) pulmonary aspergillosis occurred and despite associated intravenous antifungal therapy he died on the 5th day after clinical signs appeared (cough, low grade fever/fever, dyspnea, hemoptysis). 

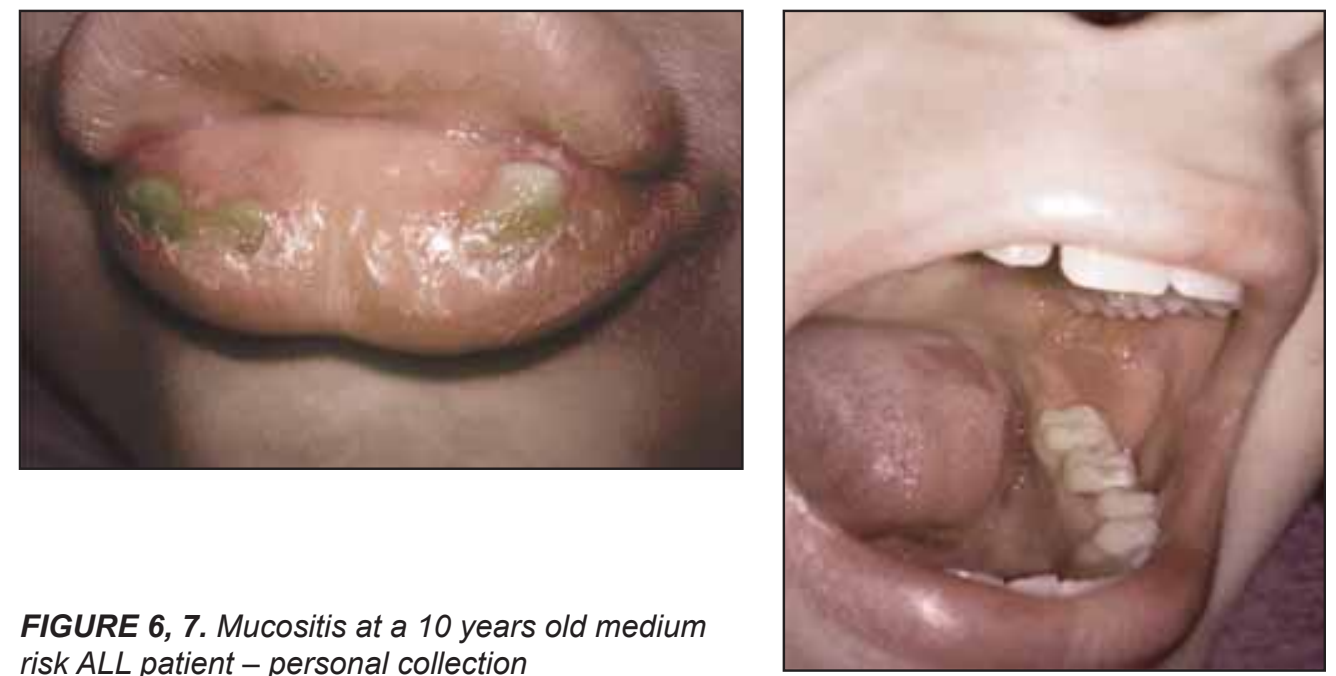

FIGURE 6, 7. Mucositis at a 10 years old medium risk $A L L$ patient - personal collection

Enteric infections occurred in 5 children with ALL and AML and consist of intense abdominal and perianal region pain, watery diarrhea with or without pathological elements, cultures did not come out positive.

Wide spectrum antibiotic treatment administered two weeks resolved these infections in all patients.

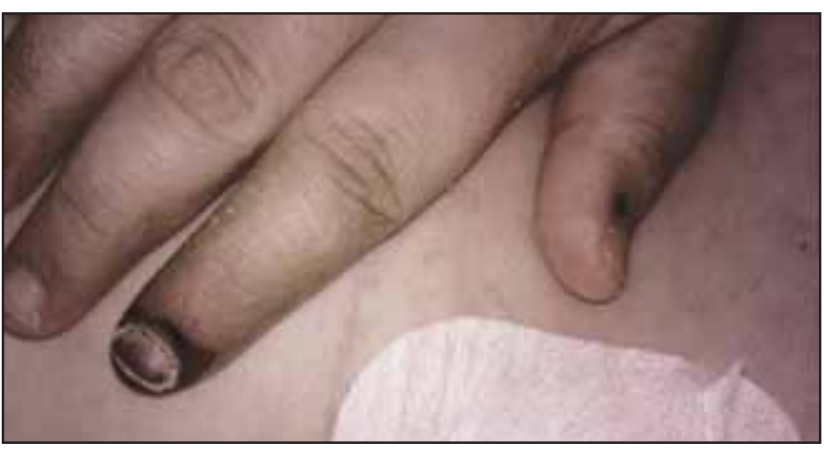

FIGURE 8. Healing panaritium 4,5 years old MR ALL patient - personal collection

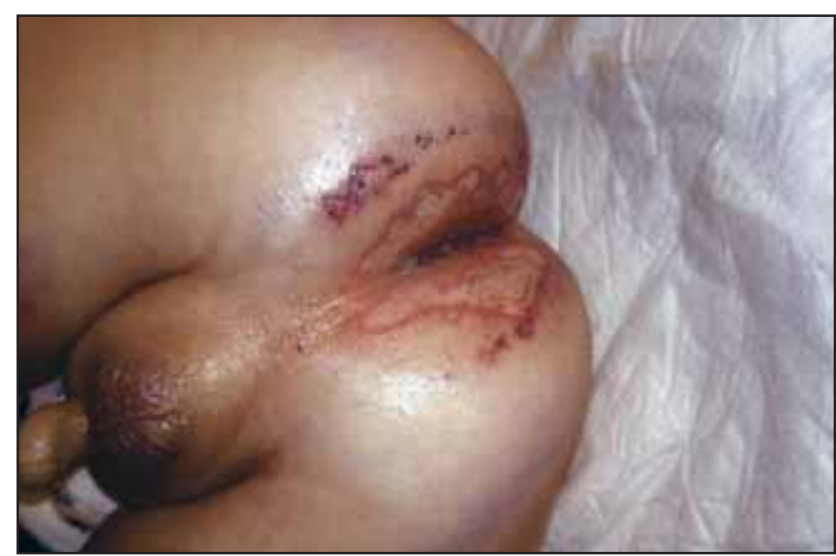

FIGURE 9. Infected intertrigo, 2 years old preB MR ALL patient - personal collection
After a cytostatic treatment in November 2015 in the middle of a rotavirus epidemic, we have identified the virus in 3 ALL patients aged 11 months, 1.5 year and 4.5 year.

Among patients hospitalized and treated four children with ALL had skin infections: panaritium (Fig. 8), skin abscesses, infected intertrigo (Fig. 9), infection above CVC (Fig. 10) and a fat obese patient presented cellulite in the breast that led to the loss of tunneled CVC.

The average duration of healing was 11 days.

In the adolescent patient with Hodgkin lymphoma during aplasia shingles was reactivated, which is why we isolated him and a day later, after we established treatment with acyclovir $80 \mathrm{mg} / \mathrm{kg}$ for a month, we released him (source of lethal infection for the remaining patients). (13)

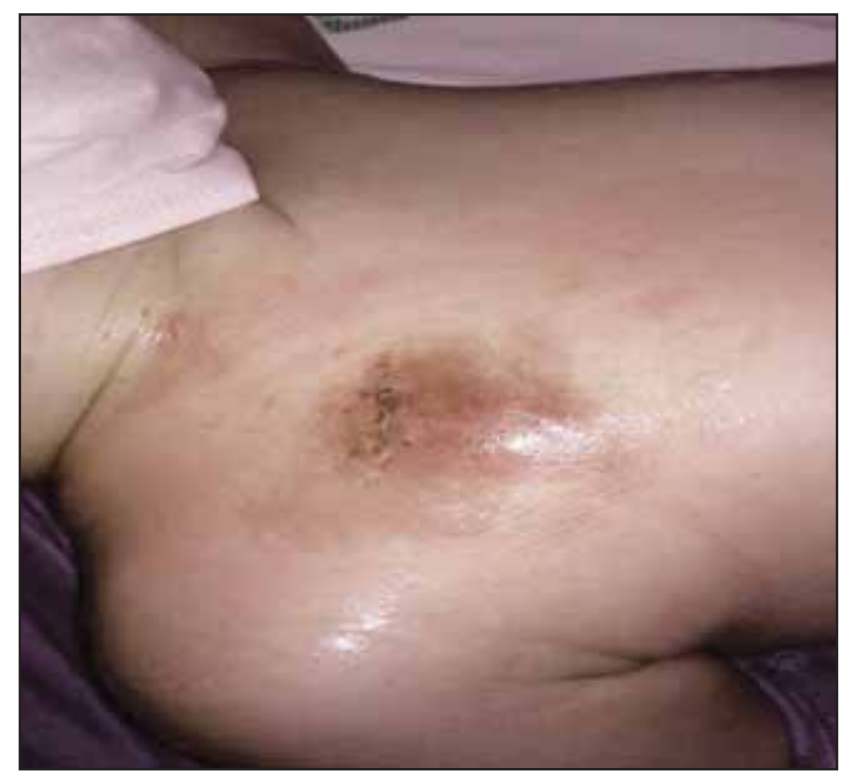

FIGURE 10. Infected CVC, 4,5 years old pre B MR ALL patient - personal collection 


\section{DISCUSSIONS}

Pathology distribution by age in our study respected data from the literature that says that ALL is most common in children aged 3-8 years and lymphomas and solid tumors affect teenager.

Infectious pathology was independent of the basic disease.

Cytopenias were corrected with packed red cells, platelets and subcutan FSCG.

Mucositis was resolved with local antiseptic treatment, painkillers, antibiotics and intravenous antifungal medications and FSCG helped leukocytes to increase during this period.

Clinical signs in bacterial pneumonia are cough, fever, dyspnoea.

Treatment consists of associated antibiotics (CG2-3, Meropenem, antistaphylococcal treatment) and immune support by intravenous immunoglobulin.

Pulmonary Aspergillosis (cough, low grade fever/fever, dyspnea, hemoptysis) is difficult to treat with a poor prognosis despite associated antifungal medication (Caspofungin, voriconazole). $(9,10)$

To prevent infection with Pneumocystis carinii all patients receive prophylaxis with trimethoprim/ sulfamethoxazole 3 years, 3 days/week $(\mathrm{V}, \mathrm{S}, \mathrm{D})$.

A feared infectious pathology is necrotisant enteritis/tiphlitis and perianal abscess that can lead to sepsis quick.

Characteristic symptoms are intense abdominal pain, diarrhea and pain during defecation.

Associate wide spectrum antibiotic treatment administered two weeks and intravenous immunoglobulins resolves the infection. (11)

Treatment of skin infections consists of washing daily the infected regions with chloramine solution, open and evacuate purulent collections, systemic antibiotics that target Staphylococcus as germ and intravenous immunoglobulin. (12)

Viral infections are extremely dangerous for immune suppressed organism, all patients who are contacts or even develop symptoms of infectious disease are isolated, and in case of varicella/zoster suspicion acyclovir therapy instituted $80 \mathrm{mg} / \mathrm{kg}$ for one month. (13)

Except for the 12 years patients with $\mathrm{Ph}$ cr. positive ALL and the 4.5 years patient with AML who associated respiratory, enteric and skin pathologies, we observed at the remaining patients a predilection for different pathologies, probably relates to their immune status urging us to step in the future to another level, studying immunological background of onco-hematology patients. (14)

\section{CONCLUSIONS}

Cancer diagnosis in advanced stages of disease decreases the prognosis for patients.

Chemotherapy treatments cause severe aplasia, which are more pronounced in children with hematologic malignancies where the bone marrow is affected, infiltrated by blasts than in case of solid tumors, where the aplasia is shorter and easier to correct.

Most clinical manifestations occurring during aplasia are of infectious origin and depend on child immunity and maternal compliance that helps maintaining required hygienic-dietary rules.

Without supportive treatment is not possible survival during cancer therapy.

Each aplasia episode can be considered real threat to the patient's life and exitus can occure by septic complications or extreme bleeding.

Infectious complications developed with individual nuances probably generated immunological.

\section{Acknowledgments}

We wish to send our special thanks to the University of Medicine and Pharmacy Targu Mures and and our Clinic staff, both nurses and physicians colleagues.

\section{REFERENCES}

1. Primic-Zakelj M. Cancer epidemiology. In Scrijvers D, Senn HJ, Mellstedt H, Zakotnk B ( eds) European Society of Medical Oncology Handbook of Cancer Prevention. Informa Healthcare 2008: 1- 28.

2. Şuteu O. Epidemiologia cancerului. In Nagy Viorica (ed) Principii de cancerologie generală.Curs pentru studenți. Editura Medicală Universitară "Iuliu Hațeganu”, Cluj-Napoca 2007: 1-24.

3. Ph. Lanzkowsky, Manual of pediatric hematology and oncologyfourth edition, 2005, 548-560

4. Nakamura, Leah, Ritchey, Michael - Current management of Wilms tumor, Current Urology Reports, Nr. 11, 58-65, 2010,

5. Hoffman R. Hematology - basic principles and practice. New York: Elsevier, 2006:cap 59-66.

6. Young N., Gerson S., High K. Clinical hematology. Philadelphia: Lipincott Williams \& Wilkins 2006: 348-402, 417-447.

7. Mitchell C., Richards S., Harrison C.J., Eden T. Long-term follow-up of the United Kingdom Medical Research Council protocols for 
childhood acute Lymphoblastic leukaemia, 1980-2001. Leukemia. 2010; 24(2):406-418. doi:10.1038/leu.2009.256.

8. Viet C.T., Corby P.M., Akinwande A., Schmidt B.L. Review of Preclinical Studies on Treatment of Mucositis and Associated Pain. Journal of Dental Research. 2014; 93(9):868-875. doi:10.1177/0022034514540174.

9. Garcia J.B., Lei X., Wierda W., et al. Pneumonia during Remission Induction Chemotherapy in Patients with Acute Leukemia. Annals of the American Thoracic Society. 2013; 10(5):432-440. doi:10.1513/ AnnalsATS.201304-0970C

10. Barkati S., Dufresne S.F., Bélanger S., et al. Incidence of invasive aspergillosis following remission-induction chemotherapy for acute leukemia: a retrospective cohort study in a single Canadian tertiary care centre. CMAJ Open. 2014; 2(2):E86-E93. doi:10.9778/ cmajo.20130062.
11. Caselli D., Paolicchi O. Empiric antibiotic therapy in a child with cancer and suspected septicemia. Pediatric Reports. 2012; 4(1):e2. doi:10.4081/pr.2012.e2.

12. Miedema K.G.E., de Bont ESJM, Elferink RFMO, et al. The diagnostic value of CRP, IL-8, PCT, and STREM-1 in the detection of bacterial infections in pediatric oncology patients with febrile neutropenia. Supportive Care in Cancer. 2011; 19(10):1593-1600. doi:10.1007/s00520-010-0987-6.

13. Forbes H.J., Bhaskaran K., Thomas S.L., Smeeth L., Clayton T., Langan S.M. Quantification of risk factors for herpes zoster: population based case-control study. BMJ : British Medical Journal. 2014; 348:g2911. doi:10.1136/bmj.g2911.

14. Swaminathan S., Klemm L., Park E., et al. Mechanisms of clonal evolution in childhood acute lymphoblastic leukemia. Nature immunology. 2015; 16(7):766-774. doi:10.1038/ni.3160 